\begin{tabular}{|c|c|}
\hline Title & $\begin{array}{l}\text { Spatial arrangement of speckle multiplexed holograms using directional control of shift selectivity with holographic } \\
\text { diffuser }\end{array}$ \\
\hline Author(s) & Takahashi, Shuji; Bunsen, Masatoshi; Furuta, Hirosuke; Okamoto, A tsushi \\
\hline Citation & $\begin{array}{l}\text { TENCON 2010: } 2010 \text { IEEE Region } 10 \text { Conference, 1625-1628 } \\
\text { https://doi.org/10.1109//ENCON.2010.5686032 }\end{array}$ \\
\hline Issue Date & $2010-11$ \\
\hline Doc URL & http:/hdl.handle.net/2115/49920 \\
\hline Rights & $\begin{array}{l}\text { () } 2010 \text { IEEE. Reprinted, with permission, from Takahashi, S., Bunsen, M., Furuta, H., Okamoto, A., Spatial } \\
\text { arrangement of speckle-multiplexed holograms using directional control of shift selectivity with holographic diffuser, } \\
\text { TENCON 2010: } 2010 \text { IEEE Region } 10 \text { Conference, Nov. } 2010 \text {. This material is posted here with permission of the } \\
\text { IEEE. Such permission of the IEEE does not in any way imply IEEE endorsement of any of Hokkaido University } \\
\text { products or services. Internal or personal use of this material is permitted. However, permission to reprint/republish this } \\
\text { material for advertising or promotional purposes or for creating new collective works for resale or redistribution must } \\
\text { be obtained from the IEEE by writing to pubs permissions@ ieee.org. By choosing to view this document, you agree to } \\
\text { all provisions of the copyright laws protecting it. }\end{array}$ \\
\hline Tyре & proceedings(author version) \\
\hline File Information & TENCON2010_1625-1628.pdf \\
\hline
\end{tabular}

Instructions for use 


\section{Spatial Arrangement of Speckle-multiplexed Holograms using Directional Control of Shift Selectivity with Holographic Diffuser}

\author{
Shuji Takahashi, Masatoshi Bunsen, Hirosuke Furuta \\ Graduate School of Engineering, Fukuoka Univ. \\ Fukuoka, Japan \\ td092003@cis.fukuoka-u.ac.jp
}

\author{
Atsushi Okamoto \\ Graduate School of Information Science and Technology, \\ Hokkaido Univ. \\ Sapporo, Japan \\ ao@optnet.ist.hokudai.ac.jp
}

\begin{abstract}
We propose a method for directional control of the shift selectivity of the speckle-multiplexed holograms using the holographic diffuser whose surface profile varies with the orthogonal direction. The method improves the tolerance of the spatial multiplexed holograms for the repositioning error in the holographic storage. In addition, we utilize the nature that the shift selectivity in the propagation direction of the reference beam is intrinsically relaxed, and experimentally implement the spatial arrangement of the multiplexed holograms in both the "controlled" and the "intrinsically relaxed" directions. We show that the high quality output is obtained using our method by the measurement of the BER of the data-pages that are retrieved from the spatially arranged multiple holograms.
\end{abstract}

Keywords- holographic data storage; speckle multiplexing; shift selectivity; holographic diffuser; spatial multiplexing

\section{INTRODUCTION}

In recent years, high quality data is flowing by development of broadband communication and digital broadcast, so new data storage with larger data capacity than existing one to store the data is needed. However, it is difficult to achieve larger capacity of optical disc and hard disc. Therefore holographic storage has attracted attention as next generation optical memory. Holographic storage uses data-page in which binary data is arranged in a matrix as documentary information. This enables us to get high transfer rate by recording and reading of the bit information in block. The data-page is displayed on spatial light modulator (SLM), and laser light through there. The light bearing data-page image is called object beam. The object beam intersects and interferes with reference beam in the holographic medium. The reference beam acts the recording beam and the reading beam. The interference pattern induces refractive-index grating as a hologram in the holographic medium. Hologram can be multiplexed in the same region by utilization of Bragg selectivity. Multiplexed hologram is also arranged spatially in the holographic medium. This is called spatial multiplexing. The capacity of the holographic storage is determined by recording density and the number of recording spot. There are many hologram multiplexing methods, such as angular, wavelength and phase-code. The speckle-multiplexing that uses speckled reference beam for hologram recording is especially suitable for hologram multiplexing. This recording method can be realized with simple optical system. The hologram recorded with speckled reference beam has the acute shift selectivity. The nature is suitable for increasing multiplicity [1-4].

In this paper, we propose the method for the spatial arrangement of large number of speckle-multiplexed hologram in holographic medium. When the speckle-multiplexed hologram is spatially arranged, the holographic medium changes the recording spot by the mechanical stage. Here, speckle-multiplexed hologram has the acute shift selectivity. Thus, quite high shift accuracy of the mechanical stage is required to obtain high quality output. Therefore, we propose a controlling method of shift selectivity corresponding to shift direction to ease the spatial multiplexing. This method enables us to relax the shift selectivity in a shift direction of holographic medium. We also notice that the hologram which is recorded with the speckled reference beam intrinsically has the relaxed shift selectivity along the propagation direction of the reference beam [1,5]. The hologram can be easily multiplexed and read out spatially along the "controlled" and the "intrinsically relaxed" directions by utilization of these relaxed shift selectivities. In section 2, we propose the method of directional control of sift selectivity by using holographic diffuser that has different autocorrelation of surface profile in an orthogonal direction. In this method, the high quality data can be retrieved from spatially arranged multiple holograms. In section 3 , we experimentally show that the shift selectivity in orthogonal plane of the propagation direction of the reference beam can be directionally controlled by use of the holographic diffuser. We also confirm that the shift selectivity along the propagation direction of the reference beam is intrinsically relaxed. Furthermore we show that the high quality data can be retrieved from spatially arranged multiple holograms by measurement of the bit error rate (BER) of the reconstructed data-page. In section 4, we conclude this research and present the future tasks. 


\section{DIRECTIONAL CONTROL OF SIFT SELECTIVITY OF SPECKLE-MULTIPLEXED HOLOGRAM BY USE OF HOLOGRAPHIC DIFFUSER}

In this section, we propose a method of the directional control of shift selectivity of speckle-multiplexed hologram by use of holographic diffuser that has different autocorrelation of the surface profile in orthogonal directions. As shown in Fig. 1, holograms are multiplexed by the horizontal shift of the diffuser which is arranged in optical path of the reference beam. The regions of the multiplexed hologram are spatially arranged in a vertical direction by the vertical shift of the holographic medium. Conventionally, we have used the diffuser which has same diffusion angle in all directions. In the case, the hologram has the acute shift selectivity in all directions. In this paper, we use the holographic diffuser whose surface profile vary with the orthogonal directions. The holographic diffuser has different diffusion angle in orthogonal directions by the surface profile. In Fig. 2, we show the optical microscopic image of the surface of the holographic diffuser. We understand that the holographic diffuser has the fine asperity and the coarse asperity in orthogonal directions. We measured the depth value of asperity along several lines in the directions of the fine asperity and the coarse asperity. We calculated the autocorrelation functions from the measured depth values, and averaged the autocorrelation functions. In Fig. 3, we show the averaged autocorrelation functions of the surface profile of the holographic diffuser. The autocorrelation distance in the direction of the fine asperity is small, although the autocorrelation distance in the direction of the coarse asperity is large. The reference beam diffused by the holographic diffuser is collected at the recording spot in holographic medium by the 4-f imaging system. This ideally ensure that the phase distribution of speckle pattern behind the diffuser corresponds approximately to the phase distribution of speckle pattern at the recording spot. We also expect that the phase distribution of speckle pattern behind the diffuser is proportional to the surface profile of the diffuser. Furthermore the shift selectivity of speckle-multiplexed hologram is proportional to the autocorrelation distance of the speckle pattern. Therefore, the acute shift selectivity and the relaxed shift selectivity appear in the directions of the fine asperity and the coarse asperity respectively. This enables us to feasibly get high quality output, while remaining high hologram multiplicity. Furthermore, the speckled reference beam also has the relaxed shift selectivity in the direction of the propagation direction of the reference beam because of the autocorrelation distance along the direction is intrinsically large. Utilizing the nature, the multiplexed holograms with the relaxed shift selectivity can be arranged spatially in the propagation direction of the reference beam in addition to the vertical direction. Combining these directions, we achieve spatially arrangement of large number of the multiplexed hologram in the holographic medium.

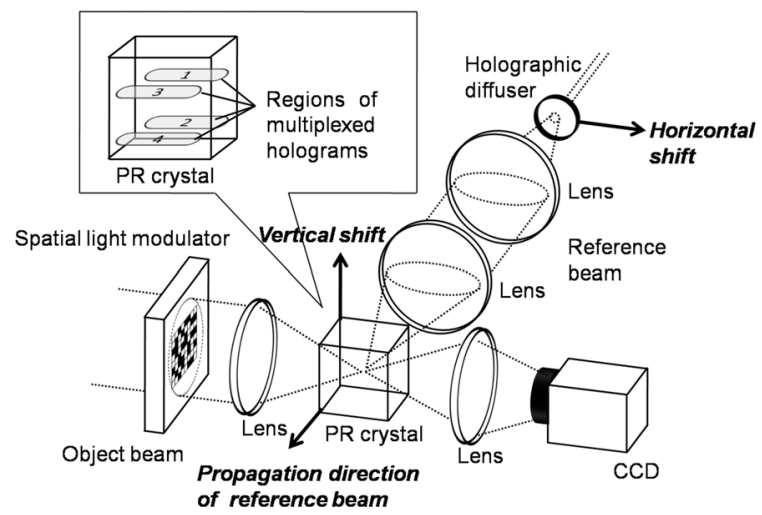

Figure 1. Optical configuration for speckle and spatial multiplexing of holograms.

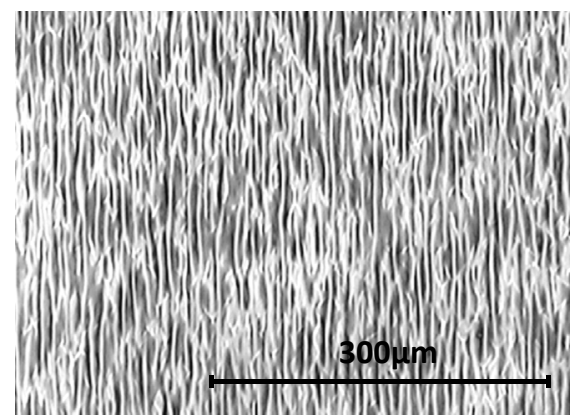

Figure 2. Optical microscopic image of the holographic diffuser surface.

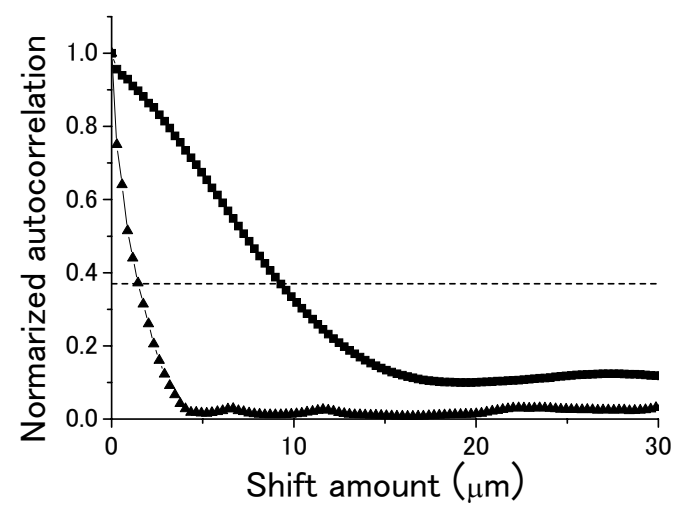

Figure 3. Averaged autocorrelation function of the surface profile of the holographic diffuser for horizontal ( $\boldsymbol{\Delta})$ and vertical ( $\boldsymbol{\square})$ shift amounts. The dashed line represents $1 / \mathrm{e}$ value.

\section{EXPERIMENT}

We show the experimental setup in Fig. 4. We use the frequency doubled $\mathrm{Nd}: \mathrm{YVO}_{4}$ laser whose wavelength is 532 nm. We use a $\mathrm{Fe}: \mathrm{LiNbO}_{3}$ crystal with the dimensions of $10 \times 10 \times 25 \mathrm{~mm}^{3}$ as the holographic medium. The data-page containing $50 \times 80$ random binary bits with the $1 / 2$ modulation code is displayed on the liquid crystal spatial light modulator (LCSLM) with $800 \times 600$ pixels. The object beam is collected at the recording spot in holographic medium by the 4-f imaging system which is constructed of $\mathrm{L}_{3}$ and $\mathrm{L}_{4}$. The reference beam is diffused by the diffuser which is arranged on the optical path 
of the reference beam. The diffused reference beam is collected at the recording spot in holographic medium by the 4-f imaging system which is constructed of $\mathrm{L}_{5}$ and $\mathrm{L}_{6}$. The object beam and the reference beam intersect at a right angle and interfere in the crystal. Then the data-page is recorded as the hologram in the crystal. The data-page image is read out from the hologram by irradiation of the reference beam. The retrieved data-page images are captured by the charge coupled device (CCD) camera. In this experiment, the diffuser is mounted on the piezo-driven X-stage. As the diffuser, we use the holographic diffuser which is explained in section 2. The direction of the smaller autocorrelation distance of the holographic diffuser surface is set in the horizontal direction and the direction of the larger autocorrelation distance of the holographic diffuser surface is set in the vertical direction. The crystal is mounted on the stepping motor driven Y-and Z-stage. In the case of the hologram multiplexing in same region, the holographic diffuser is translated by the X-stage in the horizontal direction. When the holograms are spatially multiplexed, the crystal is translated by the Y-stage and the Z-stage in the propagation direction of the reference beam and the vertical direction respectively.

At first, we measure the shift selectivity of the hologram recorded by using the holographic diffuser for the shift amounts. Fig. 5 shows the normalized diffraction efficiency for the amounts of the horizontal shift of the diffuser and vertical shift of the crystal. Although the shift selectivity in the horizontal direction remains acute, the shift selectivity in the vertical direction is relaxed. As we explain in section 2, the autocorrelation of the surface profile of the diffuser is proportional to the shift selectivity of the speckle-multiplexed hologram by 4-f imaging system. As expected, the shift selectivity in Fig. 5 is relaxed in the direction of larger autocorrelation distance of the holographic diffuser surface shown in Fig. 3. However, the degree of alleviation of the autocorrelations in Fig. 3 is different from the degree of alleviation of the $1 / \mathrm{e}$ distance of normalized diffraction efficiencies in Fig. 5 because the 4-f imaging system worked nonideally. The cause of this difference is that the horizontal width of the diffused reference beam exceeds the diameter of the lens of the 4-f imaging system.

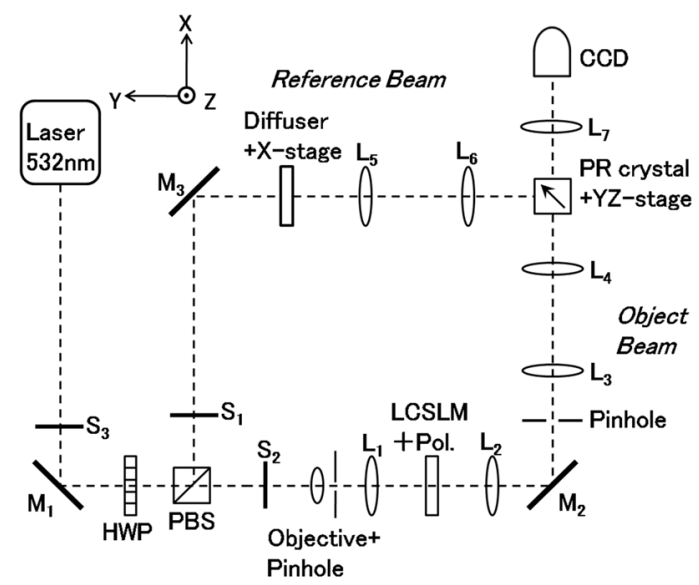

Figure 4. Experimental setup. M: mirror, L: lens, HWP: half wave plate, PBS: polarizing beam splitter, and S: shutter. The diffuser is mounted on the piezo-driven $\mathrm{X}$-stage and $\mathrm{Fe}: \mathrm{LiNbO}_{3}$ crystal is on the stepping motorized YZ-stages.
Fig. 6 shows the normalized diffraction efficiency of the shift amount in the direction of the propagation direction of the reference beam. We understand that the shift selectivity in the direction of the propagation direction of the reference beam is intrinsically relaxed without control of shift selectivity. We spatially arrange the multiplexed holograms in the Y- and Zdirections utilizing the "intrinsically relaxed" and "controlled" shift selectivities, respectively. The 30 multiplexed holograms are spatially arranged at two spots in Y- and Z-directions, respectively; the total hologram multiplicity is 120 . Fig. 7 shows the bit error rates (BERs) of the data-pages which are retrieved from the 120 multiplexed holograms. The average BERs of the multiplexed holograms in each spot are $0.57 \times 10^{-2}$, $0.60 \times 10^{-2}, 0.38 \times 10^{-3}$ and $0.38 \times 10^{-3}$. We evaluate the result as applicative value, and BER will be able to be reduced considerably by the error correcting code.

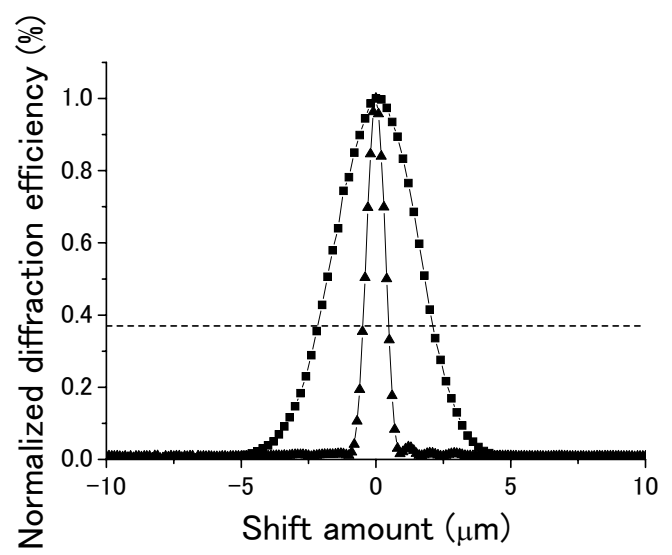

Figure 5. Normalized diffraction efficiency of the hologram recorded by speckled reference beam for horizontal $(\boldsymbol{\Delta})$ and vertical (ロ) shift amounts. The dashed line represents 1/e value.

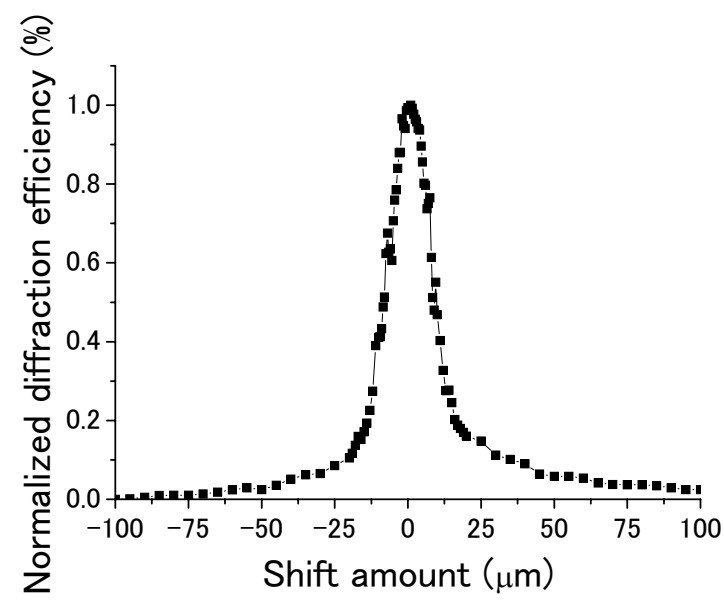

Figure 6. Normalized diffraction efficiency of the hologram recorded by speckled reference beam for the shift amount in the propagation direction of the reference beam. 


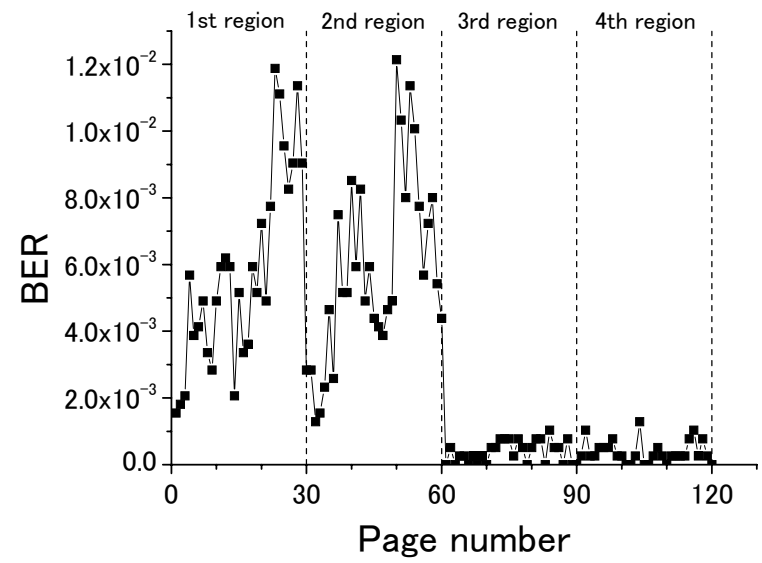

Figure 7. BERs of 120 spatially arranged multiple holograms.

\section{CONCLUSION}

To ease spatial arrangement of the speckle-multiplexed holograms, we have proposed the method of the directional control of the shift selectivity of the speckle-multiplexed holograms using the holographic diffuser whose surface profile varies with the horizontal direction and the vertical direction. We have experimentally shown that the shift selectivity can be directionally controlled using the holographic diffuser. We have also confirmed experimentally that the shift selectivity in the propagation direction of the reference beam is intrinsically relaxed. We have spatially arranged the multiplexed holograms utilizing the "intrinsically relaxed" and the "controlled" shift selectivities, and we have measured the BER of the data-pages retrieved from the spatially arranged multiple holograms. In this paper, the shift selectivity of the propagation direction of the reference beam has not been controlled, although we have utilized the intrinsically relaxed shift selectivity in the propagation direction of the reference beam. Therefore the alleviation amount of the shift selectivity is improperly large, and the clearance between multiplexed holograms in the propagation direction of the reference beam was much larger than the hologram size. For the future study, we will investigate the control of the shift selectivity in the propagation direction of the reference beam by changing the focal length of the lens in 4-f imaging system.

\section{ACKNOWLEDGMENT}

The authors appreciate Dr. H. Sueyoshi in Fukuoka IST for technical support for the measurement of the surface profile of the holographic diffuser.

\section{REFERENCES}

[1] V. Markov, J. Millerd, J. Trolinger, and M. Norrie, "Multilayer volume holographic optical memory" Opt. Lett. 24 (1999) 265.

[2] Y. H. Kang, K. H. Kim, and B. Lee, "Volume hologram scheme using optical fiber for spatial multiplexing” Opt. Lett. 22 (1997) 739.

[3] Y. H. Kang, K. H. Kim, and B. Lee, ” Angular and speckle multiplexing of photorefractive holograms by use of fiber speckle patterns" Appl. Opt. 37 (1998) 6969.

[4] M. Bunsen and A. Okamoto, "Theoretical and experimental studies of hologram multiplexing that uses a random wave front generated by photorefractive beam fanning" Appl. Opt. 44 (2005) 1454.

[5] H. Yamatsu, S. Baba, and N. Kihara, "Shift Selectivity and Hologram Playback Margin Dependence on the Average Speckle Size of a Randomly Phase Encoded Reference Beam” Jpn. J. Appl. Phys. 43 (2004) 4949. 